\title{
Ressources végétales et préférences sociales en milieu rural sénégalais
}

\author{
Mamadou Diop ${ }^{1}$ \\ Bienvenu SAMBou ${ }^{1}$ \\ Assane GoudIABY ${ }^{1}$ \\ Idrissa GUIRO ${ }^{1}$ \\ Fatimata NIANG-DIOP ${ }^{1}$ \\ ${ }^{1}$ Université Cheikh Anta Diop de Dakar \\ Faculté des sciences et techniques \\ Institut des sciences \\ de l'environnement \\ BP 5005, Dakar-Fann \\ Sénégal
}

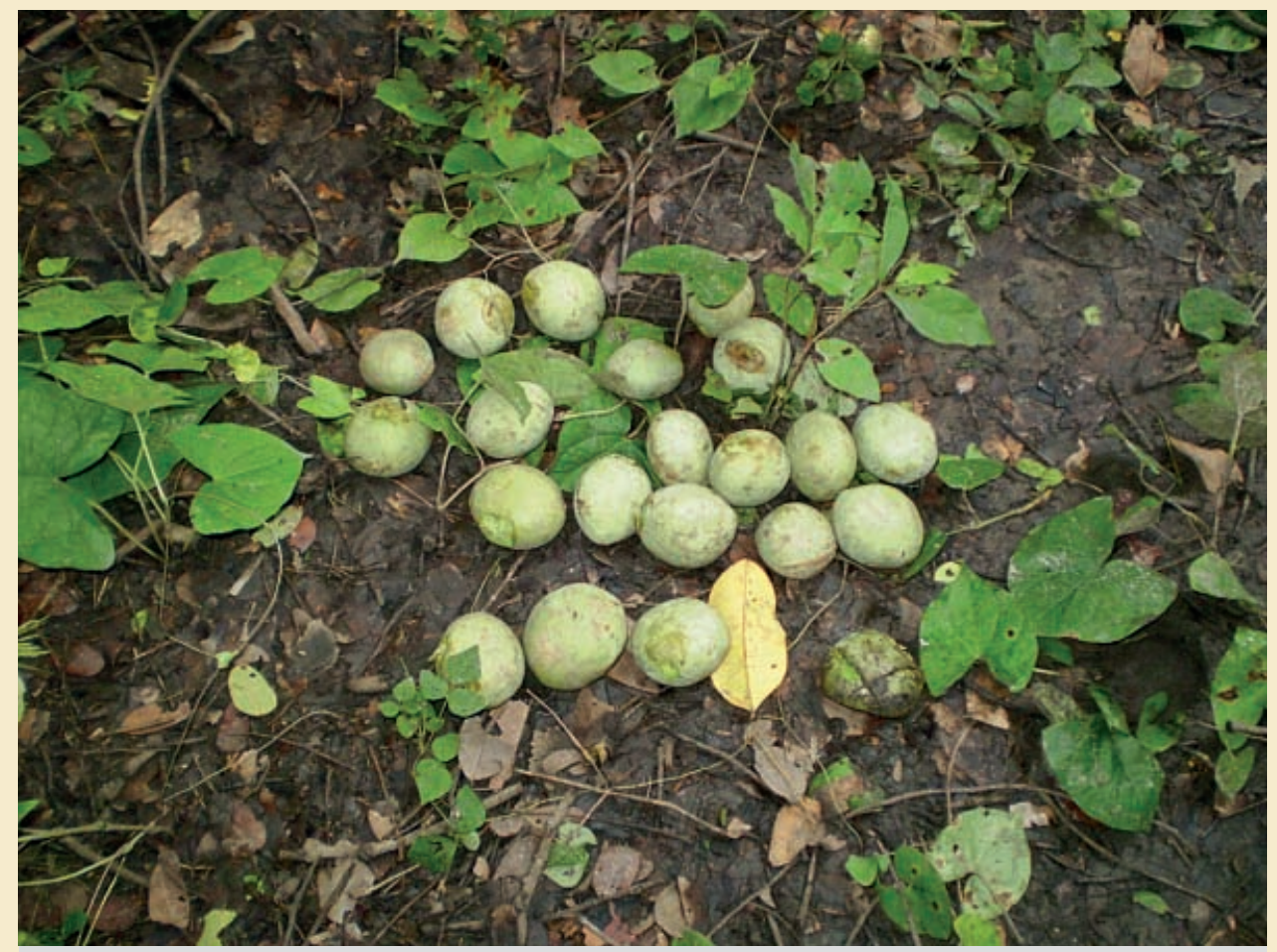

Photo 1.

Fruits de Detarium senegalensis. Le jus de ce fruit est très apprécié

au Sénégal et est très présent lors des fêtes et des cérémonies traditionnelles. Photo M. Diop. 


\section{RÉSUMÉ}

\section{RESSOURCES VÉGÉTALES ET PRÉFÉRENCES SOCIALES EN MILIEU RURAL SÉNÉGALAIS}

La connaissance des préférences des populations rurales en ressources végétales forestières est une donnée importante pour la définition des politiques de gestion et de conservation des ressources naturelles et pour la mise en œuvre des plans d'aménagement forestier. Cette étude ethnobotanique, essentiellement qualitative, a permis, grâce à l'utilisation de la matrice de classification préférentielle par paire, d'identifier les espèces végétales préférées des populations riveraines de la forêt classée de Patako (région administrative de Fatick, Sénégal). Les résultats de l'étude montrent que, sur 73 espèces ligneuses répertoriées, les préférences des populations locales portent sur 34 espèces. Chez les hommes, la possibilité d'en tirer du bois de service est le critère le plus déterminant dans le choix des espèces, suivie des possibilités d'utilisation en phytothérapie, dans l'alimentation du bétail et dans l'alimentation humaine. Chez les femmes, les préférences sont plutôt motivées par les possibilités d'approvisionnement en fruits et en bois d'énergie. La possibilité d'en tirer des remèdes contre les inconvénients de la maternité, les maladies infantiles, le paludisme et l'hypertension artérielle a été de même déterminante dans le choix des espèces chez les femmes. Cordyla pinnata et Parkia biglobosa, malgré leur régression quantitative significative, sont les deux espèces davantage préférées dans cette partie méridionale du Saloum. Même si, au plan méthodologique, le choix des participants peut constituer une limite, les résultats de l'étude pourront aider à améliorer les politiques de restauration du couvert végétal, surtout en zone de terroir.

Mots-clés : classification préférentielle, ethnobotanique, forêt classée de Patako, Sénégal.

ABSTRACT

\section{PLANT RESOURCES AND SOCIAL PREFERENCES IN RURAL ENVIRONMENT WITHIN SENEGAL}

Understanding preferences in rural populations regarding forest plant resources is an important factor for the definition of natural resource management and conservation policies and the implementation of forest management plans. This primarily qualitative ethnobotanical study involving a pairwise preferential classification matrix, was conducted to identify the plant species preferred by populations living near to the Patako listed forest (Fatick district, Senegal). The study results show that the local population tends to prefer 34 out of the 73 woody species listed. Among men, the most important criterion in their choice of species is their suitability for construction timber, followed by their possible medicinal uses in both animal and human food. Among women, preferences tend to be determined by possibilities for gathering fruits and firewood. Choices among women are also determined by possibilities for using plants as remedies for ailments during pregnancy and in young children, and against malaria and high blood pressure. Cordyla pinnata and Parkia biglobosa, despite their significant quantitative decline, are the two species mainly preferred in the southern part of the Saloum district. Although, methodologically speaking, the choice of participants could be a limiting factor, the results of this study could help to improve policies for restoring plant cover, especially in village zones.

Keywords: preferential classification, ethnobotany, Patako forest reserve, Senegal.

\section{RESUMEN}

\section{RECURSOS VEGETALES Y PREFERENCIAS SOCIALES EN EL MEDIO RURAL SENEGALÉS}

El conocimiento de las preferencias de los recursos vegetales forestales entre las poblaciones rurales es un dato importante para concretar políticas de manejo y conservación de recursos naturales y para la aplicación de planes de ordenamiento forestal. Este estudio etnobotánico, esencialmente cualitativo, ha permitido, gracias a la utilización de la matriz de clasificación preferencial por pares, identificar las especies vegetales preferidas por las poblaciones rurales colindantes con el bosque reservado de Patako (región administrativa de Fatick, Senegal). Los resultados del estudio muestran que, de las 73 especies leñosas catalogadas, las preferencias de las poblaciones locales conciernen 34 especies. Entre los hombres, la posibilidad de obtener madera de construcción es el criterio más decisivo para elegir les especies, seguido por las posibilidades de uso fitoterapéutico, la alimentación del ganado y la alimentación humana. Entre las mujeres, las preferencias están más bien motivadas por las posibilidades de suministro de fruta y leña. La posibilidad de obtener remedios contra las inconveniencias de la maternidad, las enfermedades infantiles, el paludismo y la hipertensión arterial fueron asimismo determinantes en la elección de las especies por las mujeres. Cordyla pinnata y Parkia biglobosa, a pesar de su significativa regresión cuantitativa, son las dos especies que gozan de mayor preferencia en esta parte meridional del Saloum. Aunque, a nivel metodológico, la elección de los participantes puede suponer un límite, los resultados del estudio podrán ayudar a mejorar las políticas de restauración de la cubierta vegetal, sobre todo en una zona de tierras comunales.

Palabras clave: clasificación preferencial, etnobotánica, reserva forestal de Patako, Senegal. 


\section{Introduction}

En Afrique de l'Ouest en général et au Sénégal en particulier, du fait de leurs usages multiples dans les domaines de l'alimentation (fruits, feuilles, fleurs, gomme, racine, etc.), de la construction, de l'énergie domestique et de la technologie locale (bois), de la médecine traditionnelle (feuilles, fleurs, fruits, racines, écorces, etc.) et de la magie (Bergeret, Ribot, 1990 ; Gautier, 1994 ; Sow, Anderson, 1996 ; République du SÉnÉgal, 2005a ; Theilade et al., 2007 ; Zerbo et al., 2011), les ressources végétales occupent une place très importante dans l'économie des communautés rurales.

Cependant, les formations végétales, surtout celles des zones de terroirs, connaissent une dégradation croissante (COMPAGNON, CONSTANTIN, 2000 ; FAO, 2005 ; ARIORI, OZEr, 2005 ; RÉPUBlique du SÉnÉGAL, 2005b). Cette dégradation a comme effet induit une diminution des ressources végétales. Cet effet, combiné à la pauvreté en milieu rural dont $57 \%$ des populations vivent avec moins de 392 francs Cfa (0,59 euro) par jour (RÉPUBLIQUE DU SÉNÉGAL, 2006a), augmente la pression sur les rares zones encore boisées dont l'essentiel est composé d'aires protégées. C'est le cas notamment de la forêt classée de Patako, située dans le Sud-Est de la région de Fatick.

D'une superficie de 5950 hectares (ha), cette forêt classée en 1934 est constituée d'une végétation de type savane arbustive à boisée. Sa flore est composée d'au moins 73 espèces ligneuses. Cette forêt dont les ressources sont aujourd'hui fortement sollicitées montre des signes de dégradation imputables surtout à l'absence de stratégies adéquates de gestion (SAmbou, 2004).

Pour sauvegarder les ressources de ce massif forestier, la priorité a été accordée à l'aménagement dans le cadre du projet "Tools for Management and Sustainable Use of Natural Vegetation in West Africa », financé par l'Union européenne, et qui concerne le Sénégal, le Burkina Fasso, le Bénin et le Niger. L'expérience a montré que, lorsqu'il s'agit de restauration du couvert végétal, de création de bois villageois ou d'exploitation des ressources végétales forestières, les préférences des populations locales constituent un critère important de décision (BELEM et al., 2008). Connaître ces préférences est indispensable pour parvenir aux meilleures décisions et aux meilleures interventions possibles. C'est dans ce souci que la présente étude ethnobotanique se fixe comme objectif l'identification des espèces végétales ligneuses préférées par les populations riveraines de la forêt classée de Patako, en vue de l'élaboration d'un plan d'aménagement et de gestion participatif pour une utilisation rationnelle des ressources.

\section{Zone d’étude}

L'étude a été menée dans la périphérie de la forêt classée de Patako, dans la région administrative de Fatick, département de Foundiougne, arrondissement de Toubacouta (figure 1). Avec une pluviosité de 600 à $700 \mathrm{~mm}$ par an, cette zone appartient au domaine soudanien (Collectif, 2007).

La forêt classée de Patako présente une mosaïque de types de végétation dont les plus représentés sont la savane arbustive à boisée au niveau des plateaux et la savane boisée en bordure des vallées. Dans la zone de confluence des deux vallées principales, la végétation est une mangrove haute constituée par Avicennia germinans (SAmbou, 2004). Sur les plateaux, les espèces ligneuses dominantes sont Combretum glutinosum, Pterocarpus erinaceus, Bombax costatum, Lannea acida, Cordyla pinnata, Acacia macrostachya et Ozoroa insignis (SAmBou, 2004). En bordure des vallées, les espèces dominantes sont Elaeis guineensis, Ficus congensis, Khaya senegalensis, Erythrophleum suaveolens, Afzelia africana et de nombreuses espèces lianescentes.

Dans les terroirs villageois, les espèces végétales sont en nette régression mais largement dominées par Cordyla pinnata, volontairement épargnée par les agriculteurs du fait surtout de l'utilisation des fruits dans plusieurs plats locaux et du rôle de l'espèce dans la fertilisation des terres.

Les deux communautés rurales (Keur Saloum Diané et Keur Samba Guèye) qui jouxtent la forêt avaient déjà à l'époque une population de 48133 habitants (RÉPUBLIQUE DU SÉNÉGAL, 2006b). La densité moyenne de 93 habitants

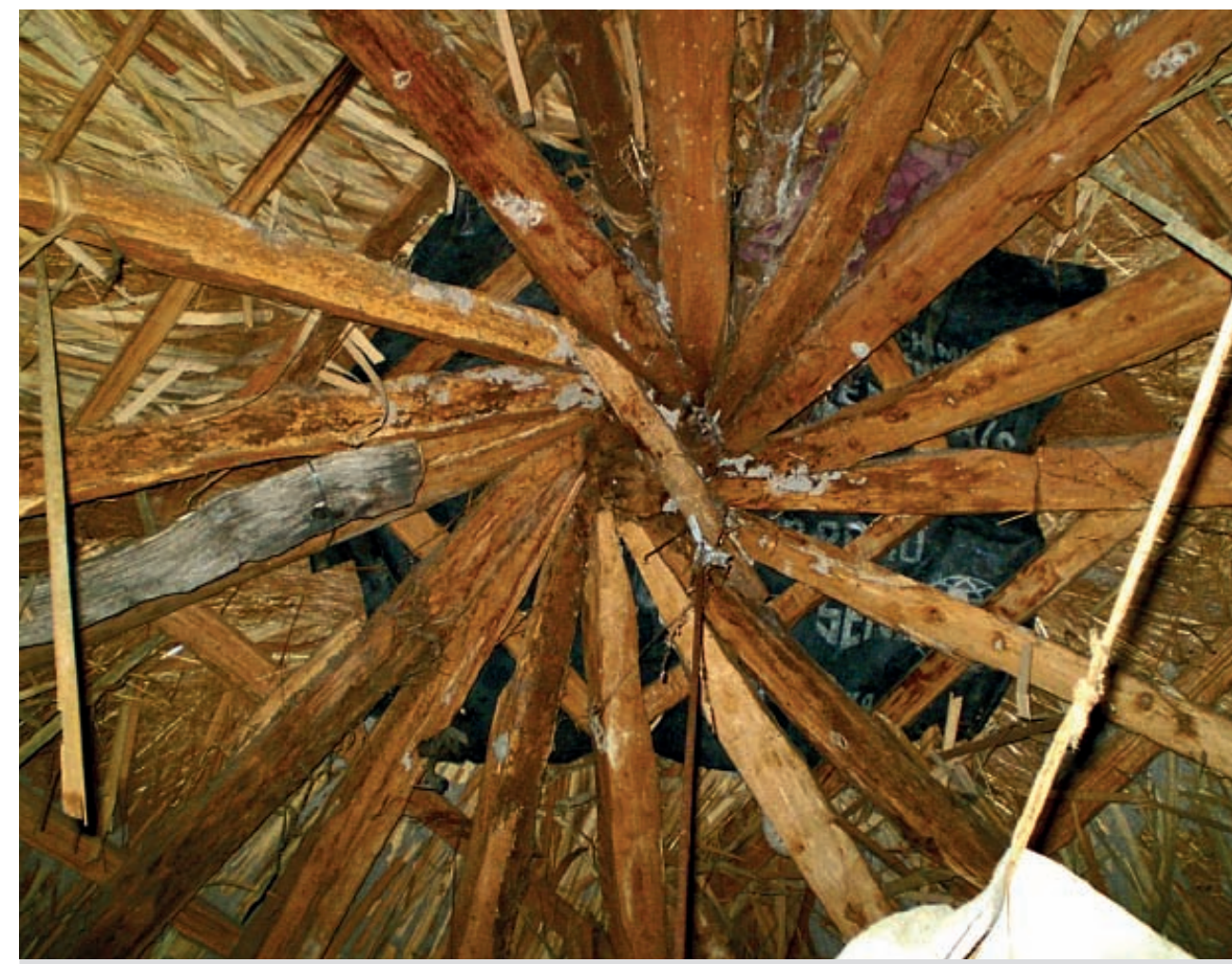

Photo 2.

Charpente d'une case en bois de Terminalia macroptera. Les populations préfèrent cette espèce car le bois est tendre et léger pour être supporté par les murs en banco. Photo M. Diop. 

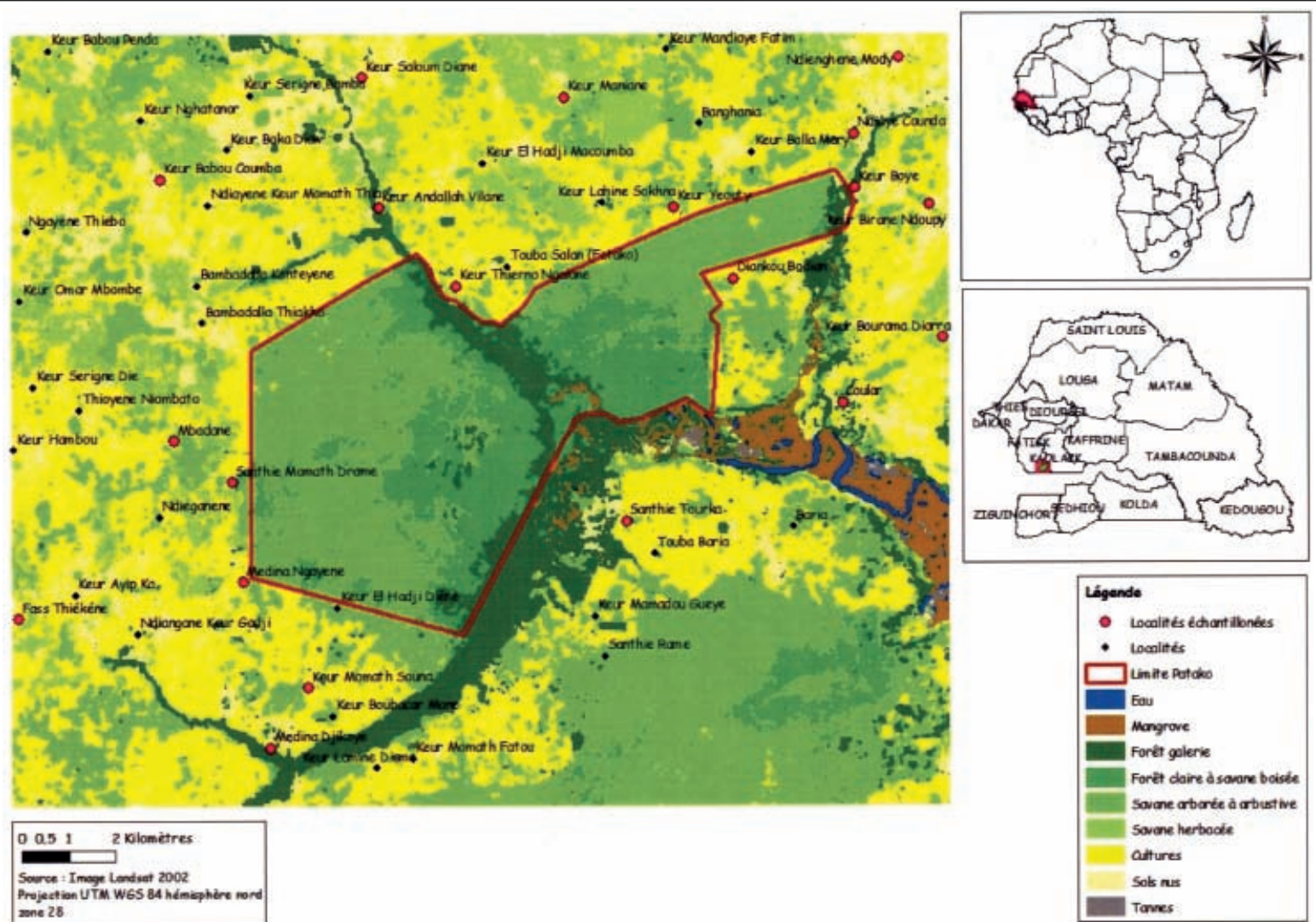

Figure 1.

Carte de localisation de la zone d'étude.

au kilomètre carré a comme conséquence une forte pression sur les ressources naturelles végétales. Cette zone compte une dizaine d'ethnies dont les plus importantes sont les Wolofs (62,5\% des habitants), les Mandingues (19\%), les Sérères, les Peuls et les Bambaras (RÉPUBLIQUE DU SÉNÉGAL, 2006b). Les activités dominantes sont l'agriculture avec comme principales productions le mil, l'arachide, le maïs, le niébé et la pastèque, ainsi que l'élevage de bovins, d'ovins et de caprins.

\section{Méthodes}

Les données ont été collectées lors d'une enquête qualitative auprès des populations riveraines de la forêt classée de Patako de juin à août 2008, dans 20 villages. La première étape de l'enquête a consisté en des entretiens informels lors d'une mission de prospection de terrain pour obtenir quelques informations sur les activités d'exploitation des ressources forestières des populations riveraines de la forêt et les principales espèces sur lesquelles portent ces activités. La deuxième étape a consisté en une recherche documentaire et un choix des outils de collecte constitués du focus group (groupe de discussion) et de la classification préférentielle par paire qui est un outil de la "Méthode accélérée de recherche participative " (GUEYE, FreudenBERgER, 1991). Ces outils ont été testés dans trois localités choisies de façon raisonnée en tenant compte de leurs similarités avec les localités étudiées, notamment la proximité avec la forêt classée, les communautés ethniques et les activités socioéconomiques. Le choix définitif a été effectué au hasard dans un rayon de cinq kilomètres en partant des limites de la forêt classée vers les zones de terroirs. Dans chaque localité choisie, un groupe de discussion a été organisé avec des hommes et un autre avec des femmes. Les groupes comprenaient entre 10 et 20 participants. Le choix de ces personnes a été fait avec l'appui du chef de village et de la présidente du groupement des femmes, en fonction de l'âge (35 ans minimum), de l'activité socioéconomique (agriculteur, éleveur, artisan), de l'appartenance ethnique et de la connaissance des ressources forestières ligneuses de la zone. Lors des groupes de discussion, les échanges ont tourné autour de trois principaux thèmes :

- ressources végétales ligneuses de la zone (richesse floristique, état, dynamique) ;

- principales espèces végétales ligneuses exploitées ;

- principales utilisations des espèces végétales ligneuses.

Dans chaque village, les participants commencent par inventorier toutes les espèces ligneuses présentes dans leur terroir, ainsi que les différents usages qu'ils en font. Cela permet d'obtenir une liste d'espèces parmi lesquelles les participants choisissent les six espèces considérées comme étant les plus importantes en fonction de leur rôle socioéconomique. Les six espèces retenues sont répertoriées dans une matrice de classification par paire, un outil permettant de déterminer les préférences. Deux espèces sont présentées aux participants, à qui il est demandé de choisir l'espèce préférée puis la raison d'un tel choix. Ce 
procédé est répété pour toutes les combinaisons d'espèces possibles. Quand le choix d'une espèce ne fait pas l'unanimité, les participants procèdent à un vote à main levée. Enfin, des scores (0 à 20) sont attribués en fonction de la fréquence et la hiérarchie des préférences est ainsi établie.

En tenant compte du score minimum (0) et du score maximum (20), il est distingué trois catégories d'espèces :

- les espèces de faible préférence $[0 ; 6]$;

- les espèces de préférence moyenne [7; 13] ;

- les espèces de grande préférence [14;20].

Les données recueillies ont été traitées avec le tableur Excel. Les noms des plantes ont été transcrits des langues locales à l'aide de documents spécialisés (BERHAUT, 1967 ; ADAM, 1970) et avec l'assistance de botanistes travaillant dans la zone. Les services d'un médecin ont permis de connaître les noms en français des maladies traitées avec les produits végétaux tirés de la forêt.

\section{Résultats}

\section{Espèces végétales préférées des femmes}

Les femmes ont porté leur choix sur $28,7 \%$ des 73 espèces ligneuses inventoriées dans cette aire protégée (SAmbou, 2004). Ces espèces, au nombre de 21, répondent essentiellement aux trois besoins fondamentaux suivants : alimentation, pharmacologie et énergie. L'exploitation des données collectées auprès des femmes met en évidence trois catégories d'espèces. La première catégorie est composée de Cordyla pinnata, Parkia biglobosa et Combretum glutinosum. Ces trois espèces jouent un rôle fondamental dans la vie de ces femmes, dans la mesure où les deux premières sont fortement sollicitées dans l'alimentation et l'économie féminine locale, alors que la troisième constitue l'espèce dont le bois est le plus exploité pour la satisfaction des besoins en énergie domestique.

Dans la deuxième catégorie, les préférences ont porté sur les espèces à fruits comestibles; il s'agit notamment de Tamarindus indica, Adansonia digitata et Detarium senegalensis (photo 1). Les deux premières sont utilisées dans des menus locaux, tandis que la troisième, en plus d'être consommée, fait l'objet d'une commercialisation. Le choix de Pterocarpus erinaceus en quatrième position s'explique surtout par ses nombreuses vertus médicinales.

À l'instar de la deuxième catégorie, la troisième est dominée par les espèces dont les fruits sont consommés par les populations locales. Il s'agit de Ficus sycomorus, Detarium microcarpum, Saba senegalensis, Neocarya macrophylla, Cola cordifolia, Ziziphus mauritiana, Lannea acida et Elaeis guineensis. Quant à Sterculia setigera, les raisons de son choix sont liées à sa résine utilisée comme liant naturel dans la préparation du couscous. Les autres espèces sont préférées principalement pour leur écorce, Ficus iteophylla et Daniellia oliveri, leur utilisation sous forme de tisane pour le petit-déjeuner, Combretum micranthum, leurs vertus médicinales, Cassia sieberiana, ou leur utilisation comme bois d'énergie, Terminalia macroptera.

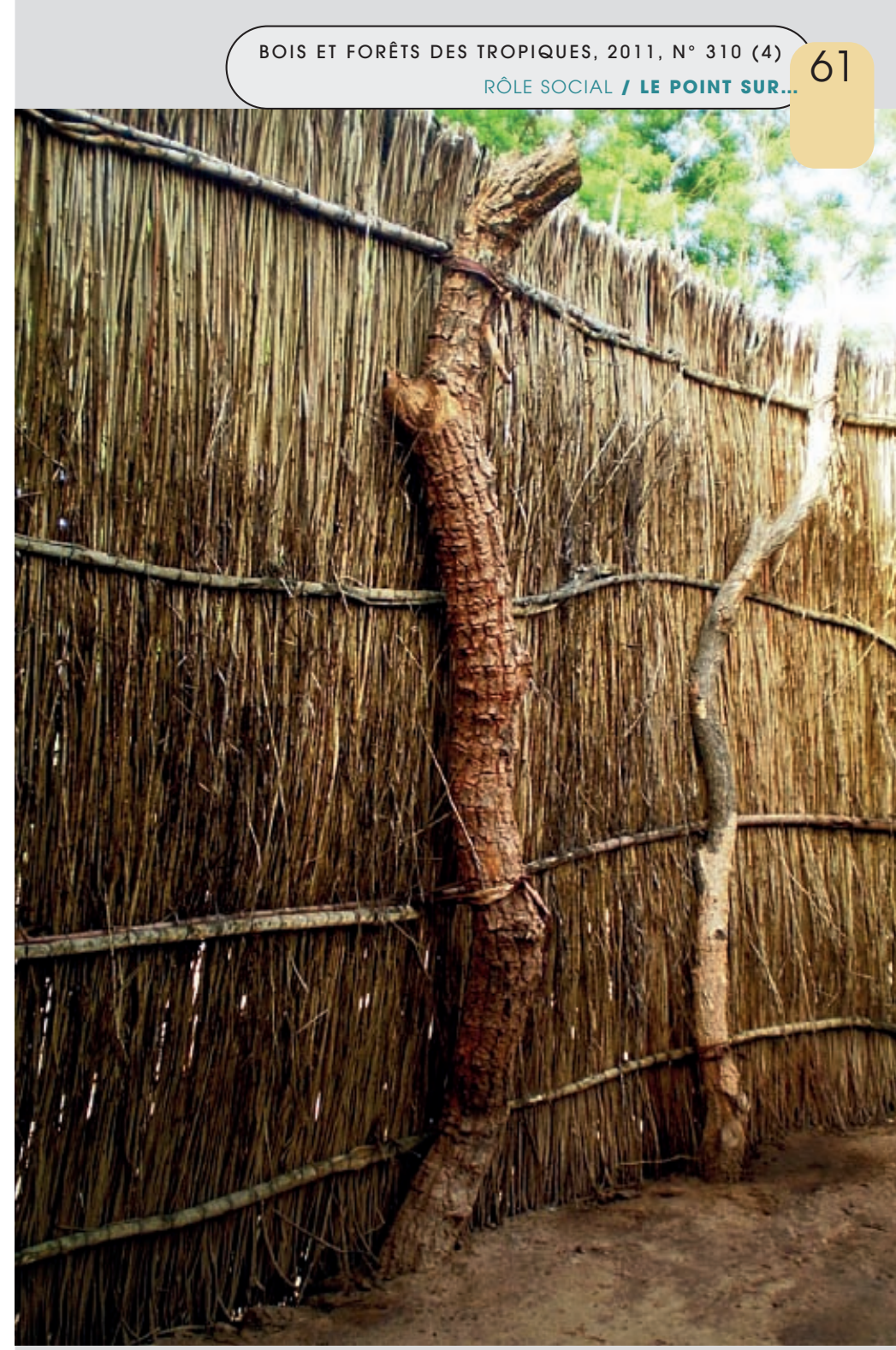

Photo 3.

Palissade supportée par des piquets en bois de Prosopis africana, espèce à bois dense non attaqué par les termites. Photo M. Diop.

\section{Espèces végétales préférées des hommes}

Sur les 73 espèces ligneuses répertoriées dans la forêt classée de Patako, les hommes ont porté leur choix sur $38,3 \%$ d'entre elles pour diverses raisons. Leur classification préférentielle est caractérisée essentiellement par une nette prédominance de trois espèces dont les scores varient entre 18 et 20 . Il s'agit de :

- Cordyla pinnata, principalement pour la place prépondérante qu'elle joue dans l'alimentation locale et l'approvisionnement en bois de service ;

- Parkia biglobosa, qui en plus de son rôle dans l'alimentation est très utilisée dans la médecine traditionnelle locale, notamment contre la conjonctivite (feuille), la dysenterie et les vers intestinaux (écorce) ;

- Pterocarpus erinaceus, pour son bois et les vertus thérapeutiques de son écorce et de ses racines contre la fatigue, l'anémie, l'ulcère et les maux de ventre. 


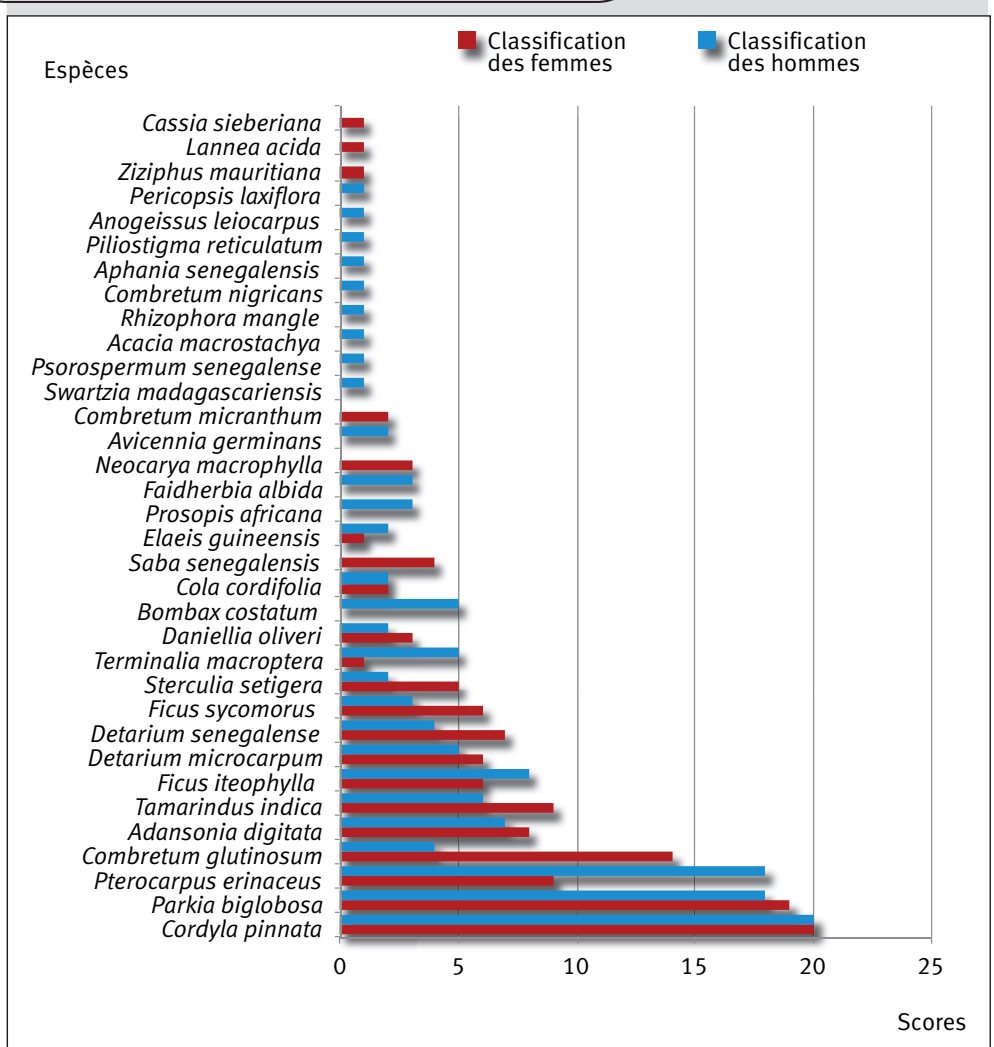

Figure 2.

Synthèse des classifications préférentielles des femmes et des hommes.

Les espèces de préférence moyenne ont été choisies pour diverses raisons dont principalement leurs vertus thérapeutiques, Ficus iteophylla, et leur utilisation dans l'alimentation, Adansonia digitata. Hormis ces cinq espèces qui composent les deux premières catégories, toutes les autres (23) ont un score relativement faible (inférieur ou égal à 6). Ces espèces ont été choisies pour leur rôle dans la construction des habitations, Acacia macrostachya, Afrormosia laxiflora, Anogeissus leiocarpus, Avicennia germinans, Bombax costatum, Combretum glutinosum, Combretum nigricans, Daniellia oliveri, Elaeis guineensis, Piliostigma reticulatum, Prosopis africana, Rhizophora mangle, Terminalia macroptera, l'alimentation, Cola cordifolia, Detarium microcarpum, Detarium senegalense, Ficus sycomorus, Tamarindus indica, Sterculia setigera, la médecine traditionnelle, Aphania senegalensis, Psorospermum senegalense, Swartzia madagascariensis, et enfin la fertilisation des terres de culture, Faidherbia albida.

La comparaison des préférences des hommes et des femmes (figure 2) a permis de mettre en évidence une convergence des préférences sur quinze espèces. C'est pour le bois et les fruits sauvages que la différence des préférences entre les femmes et les hommes est nettement perceptible. Il est apparu également que seulement quatre espèces, parmi les 34 espèces choisies, présentent un score supérieur à 10. Il s'agit de Cordyla pinnata, Parkia biglobosa, Pterocarpus erinaceus et Combretum glutinosum. Cordyla pinnata est l'unique espèce ayant atteint le score maximum (20) aussi bien chez les femmes que chez les hommes.

\section{Discussion}

Les 34 espèces ligneuses choisies par les femmes et les hommes sur un total de 73 inventoriées par SAMBOU (2004) dans cette forêt traduisent, d'une part, le niveau de connaissance des ressources forestières végétales des populations locales. D'autre part, elles révèlent l'importance de ces ressources dans l'existence des riverains de la forêt qui y satisfont l'essentiel de leurs besoins en produits ligneux et non ligneux (tableau I). Ce rôle prépondérant des ressources végétales dans le quotidien des populations locales transparaît nettement dans les raisons des choix.

Chez les hommes, la forte proportion d'espèces utilisées pour le bois de service (17 sur 28) obéit à une certaine logique, vu la nature des habitations et le rôle de l'homme dans leur édification et leur réfection. À l'instar d'autres milieux ruraux africains (Bernus, 1967 ; GANABA et al., 2004), l'habitat de type traditionnel reste étroitement lié aux ressources naturelles végétales qui fournissent l'essentiel des matériaux de construction (piquets, perches, traverses, supports, paille, chaume, cordes, etc.). Puisqu'une bonne partie de l'habitat est constituée de cases en paille ou en banco couvertes par un toit de chaume (photo 2), le tout entouré d'une clôture faite de paille et de branches, l'entretien se fait annuellement à la fin de la saison des pluies, du fait surtout du caractère précaire des matériaux. Ce qui entraîne une importante consommation de bois de service. Parmi les espèces les plus utilisées figurent Bombax costatum et Terminalia macroptera pour la faible densité de leur bois considéré comme étant le plus facile à travailler et le plus léger pour les constructions en banco. Prosopis africana, dont le bois est réputé dense et non attaqué par les termites, est généralement utilisé comme piquet pour les palissades (photo 3). Cette préférence pour les bois solides et les bois faciles à travailler a été notée chez les nomades burkinabé qui sélectionnent les plantes selon divers paramètres. Il s'agit notamment de la perception de la solidité du bois, de la résistance à la pourriture, aux termites et aux autres insectes foreurs, ainsi que de la qualité de droiture et de la facilité à travailler du bois (GANABA et al., 2004 ).

Le choix des hommes s'explique aussi par l'utilisation du bois de service dans le secteur agricole. Les manches des outils aratoires locaux sont fabriqués avec du bois de Pterocarpus erinaceus ou de Cordyla pinnata. C'est ainsi qu'une grande préférence est notée pour les racines de Pterocarpus erinaceus considérées par les populations comme plus résistantes. Dans chacune des localités proches de la forêt, existe une personne, généralement un Laobé (photo 4), qui est un bûcheron itinérant aux origines peules ayant un lien socioculturel très particulier avec l'arbre qu'il utilise pour confectionner divers produits (ustensiles de cuisine, bancs, manches à outil) destinés à la vente. Le bois de ces deux espèces est aussi utilisé pour la fabrication de jougs, éléments indispensables pour la traction bovine (labour et transport). 
En outre, si le bois de service a largement influé sur le choix des hommes, cela est aussi dû à son usage dans l'élevage et le transport. Sur les places publiques et même à l'intérieur des concessions, ce sont des troncs de Cordyla pinnata creusés latéralement qui servent d'abreuvoir pour le bétail (photo 5). Du fait de la proximité de la forêt et la présence de fauves (hyènes, chacals, etc.), les petits ruminants sont systématiquement confinés dans des enclos de bois d'un diamètre relativement important, afin d'en limiter la prédation.

Dans le transport, les arbres sont utilisés pour la fabrication de charrettes et de pirogues qui demeurent les principaux moyens de déplacement dans cette zone.

En dehors du bois de service, les préférences des hommes répondent aussi à des préoccupations d'ordre sanitaire. Le choix de plusieurs espèces a été essentiellement justifié par leurs nombreuses vertus thérapeutiques et leur forte sollicitation dans la médecine traditionnelle. Ficus iteophylla (photo 6) et Swartzia madagascariensis sont préférées pour leur usage contre la fatigue, Detarium microcarpum contre les mycoses et les diarrhées et enfin Psorospermum senegalense contre les maux de ventre. Les parties utilisées à cet effet sont respectivement l'écorce et la racine pour les deux premières espèces et la racine et la feuille pour les deux autres.

D'autres espèces ont été choisies du fait de leur utilisation dans l'alimentation ou comme aliment pour le bétail. C'est le cas de Adansonia digitata, Bombax costatum, Cola cordifolia, Cordyla pinnata, Faidherbia albida, Ficus sycomorus, Pterocarpus erinaceus, Prosopis africana, Terminalia macroptera et Sterculia setigera. Les feuilles de ces espèces sont utilisées comme fourrage pour les bovins, les ovins et les caprins en cas de retard des pluies, de mauvaise production arachidière ou de destruction du tapis herbacé par les feux de brousse. Ce faisant, les espèces que les populations exploitent le plus à cet effet sont Adansonia digitata, Bombax costatum et Pterocarpus erinaceus.

Les femmes, plus préoccupées par la famille et la maternité, ont vu leurs choix fortement influencés par les rôles qu'elles jouent dans la société rurale traditionnelle (ToBith, Cuny, 2006). C'est ainsi qu'elles ont porté leur choix sur des espèces ligneuses dont le bois est utilisé comme combustible (13 sur 21). Parmi ces espèces, Combretum glutinosum est la plus exploitée (photo 7).

L'importance de cette espèce dans la satisfaction des besoins en bois de chauffe a été notée au Burkina Faso par GANABA et al. (1998) et OuEdRAOGo (2007), au Mali par Nouvellet et al. (2003), au Bénin par LAWANı (2007), au Niger par BRondeAu (2001). En outre, l'abondance des espèces fournissant du bois de chauffe dans cette classification s'explique, d'une part, par l'utilisation de ce bois comme principale source d'énergie pour la cuisson des aliments en milieu rural sénégalais et, d'autre part, dans cette zone par le fait que les tâches ménagères qui nécessitent l'usage du bois d'énergie sont dévolues aux femmes, même si les hommes contribuent à sa collecte.

Les soins post-accouchement et les maladies infantiles ont été aussi déterminants dans le choix des femmes, de même que les soins contre l'hypertension artérielle et le paludisme. À l'exemple des hommes, la préférence de cer-

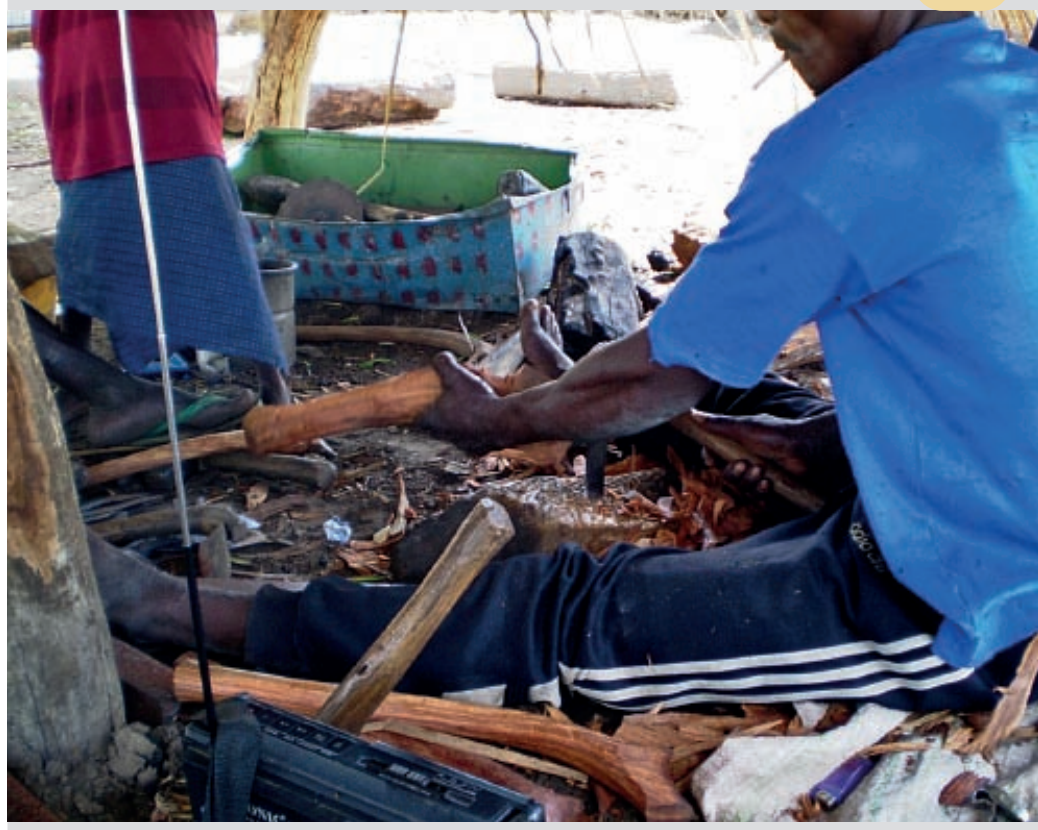

Photo 4.

Un Laobé sculptant des manches d'outils aratoires. Photo M. Diop.

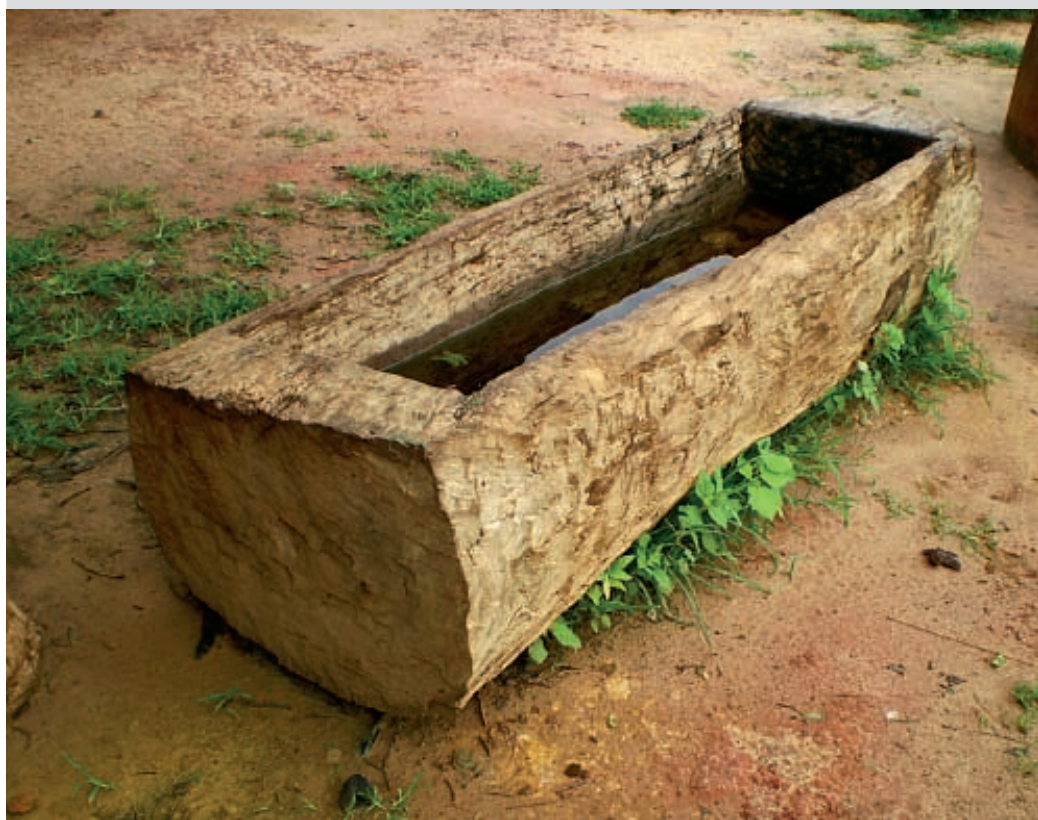

Photo 5.

Abreuvoir fait à partir d'un pied de Cordyla pinnata de gros diamètre.

Photo M. Diop.

taines espèces n'a été justifiée que par leurs vertus médicinales. Il s'agit de Pterocarpus erinaceus contre l'anémie, l'ulcère et les maux de ventre, de Ficus iteophylla contre la fatigue et les œdèmes, de Lannea acida contre les maux de ventre, l'hypertension artérielle, les parasitoses et les douleurs ou complications périnatales et enfin de Cassia sieberiana, utilisée contre les parasitoses. 


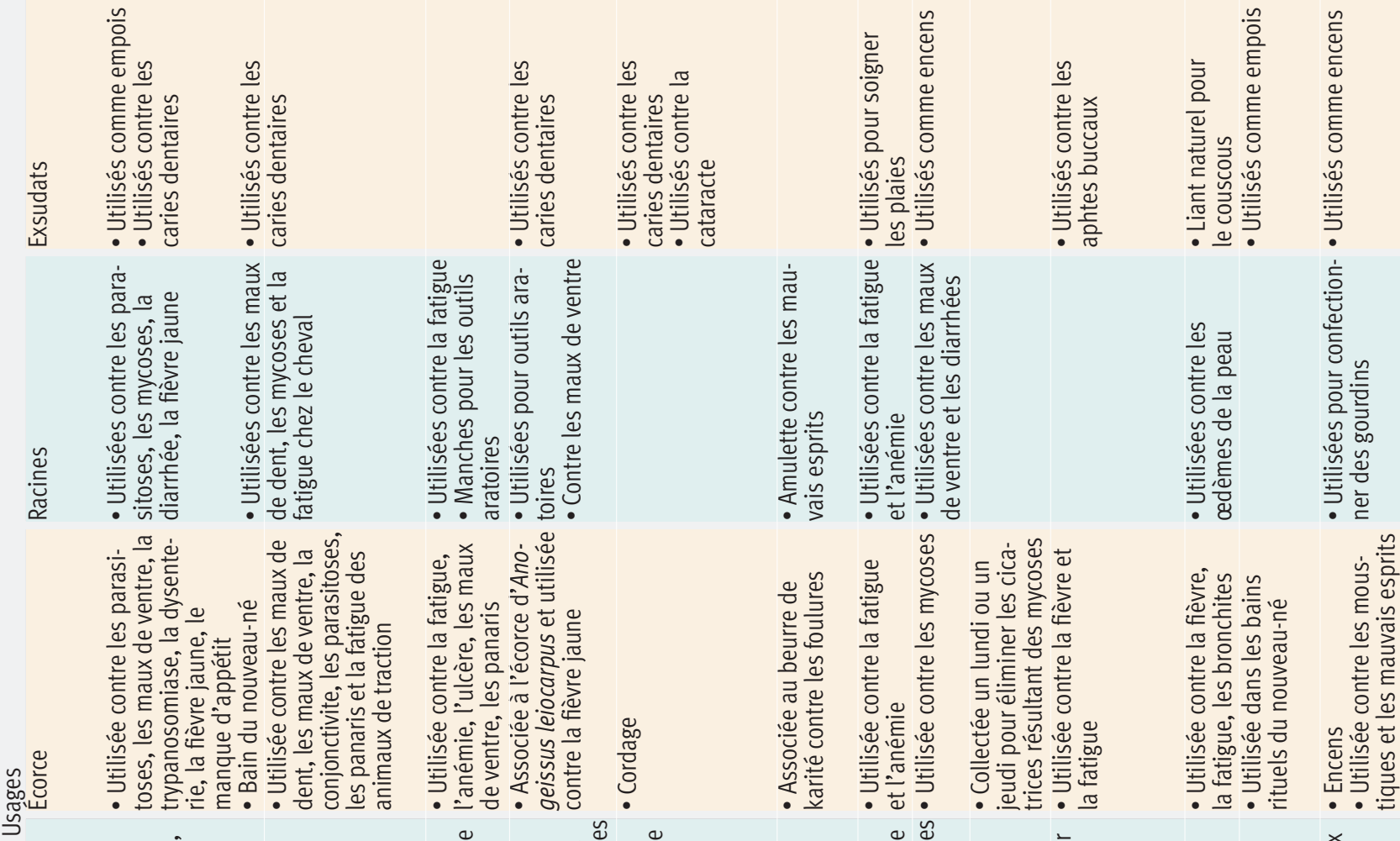

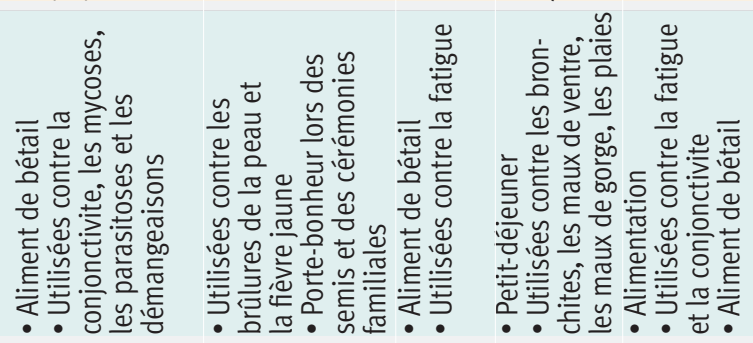

$\stackrel{\dddot{u}}{\mathrm{y}}$

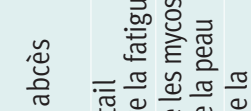

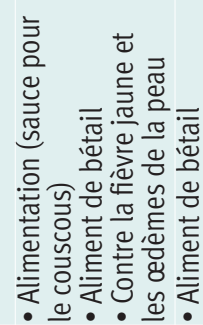

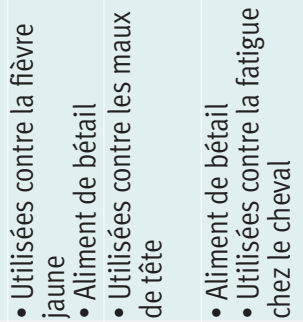

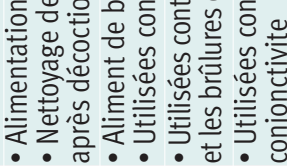

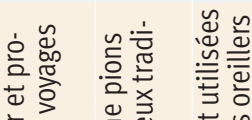

我葶

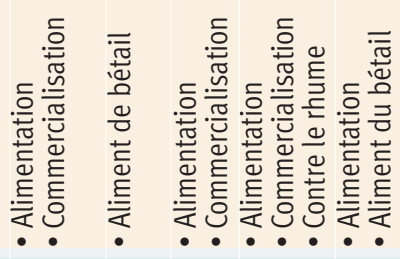

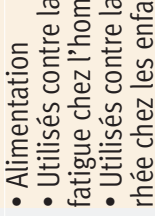

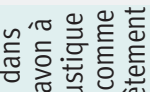

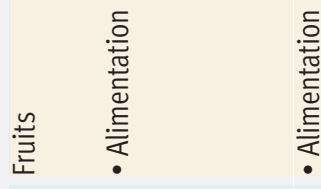

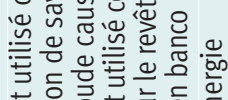

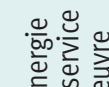

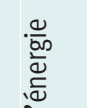

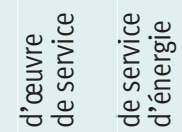

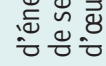

$\stackrel{n}{\circ} \quad \frac{n}{\circ} \cdot \frac{n}{\circ}$

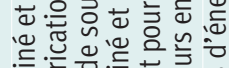

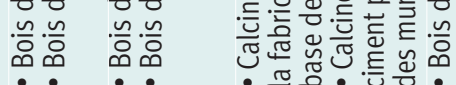

$\frac{\pi}{\sqrt{0}}$

突

产崖

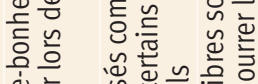

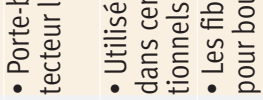

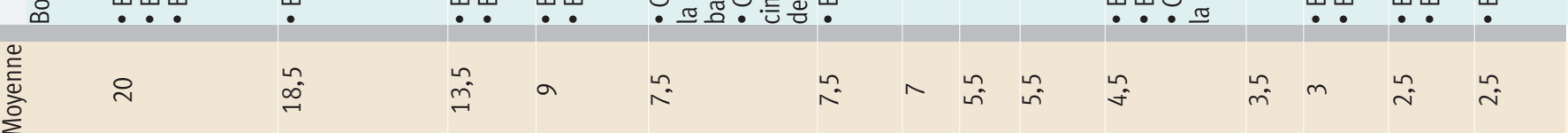

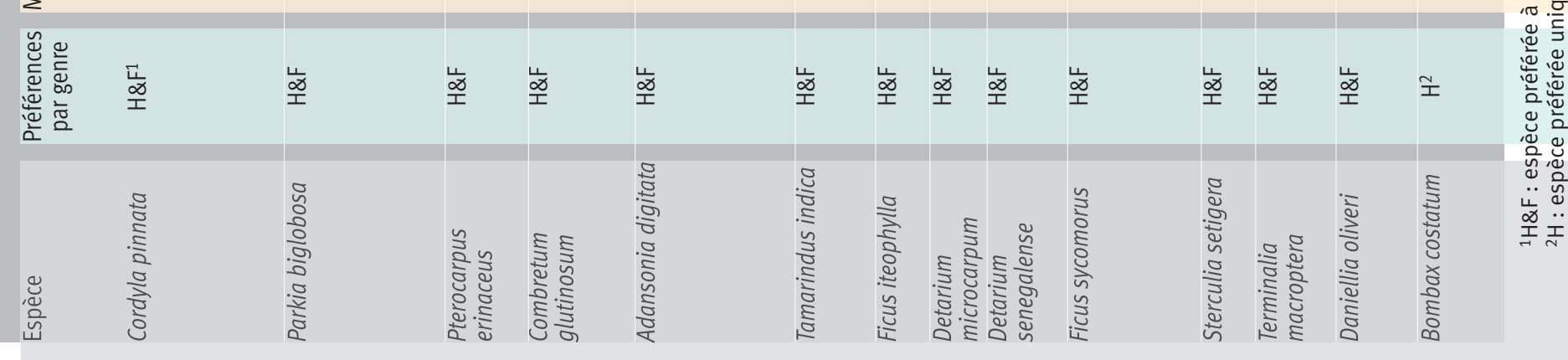




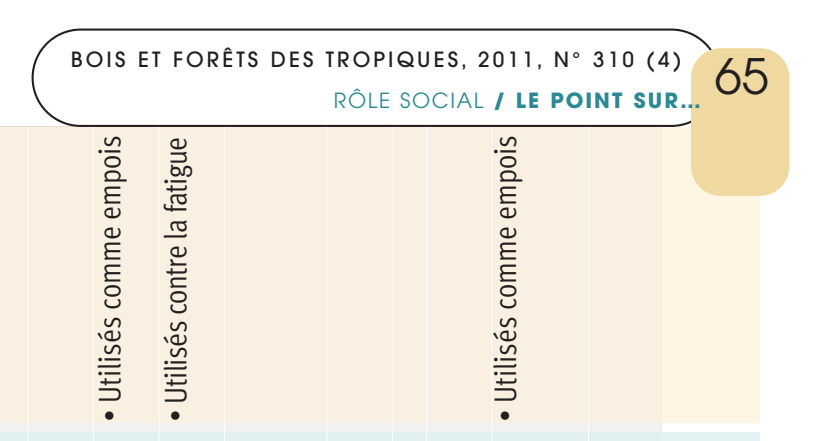

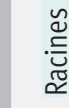
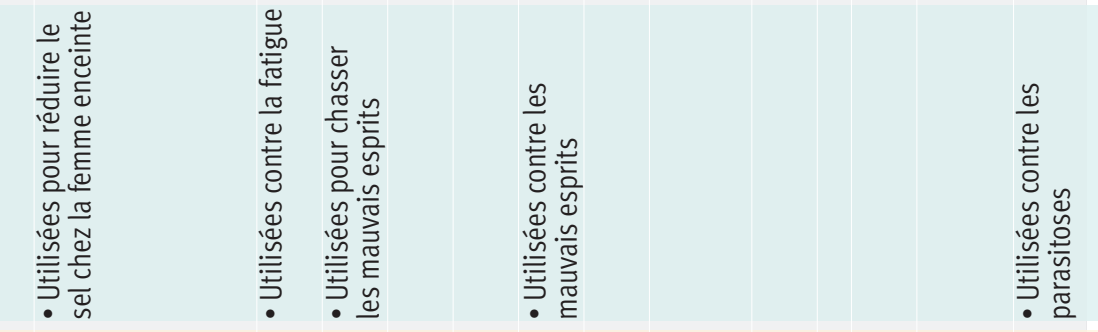
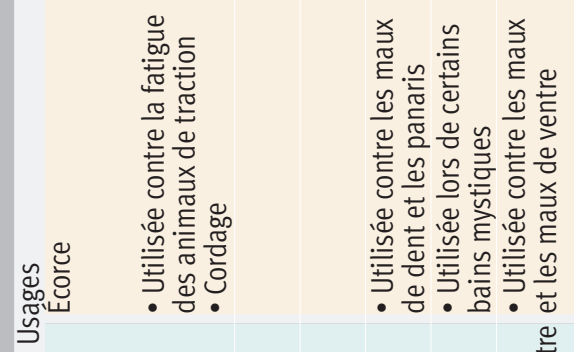

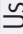

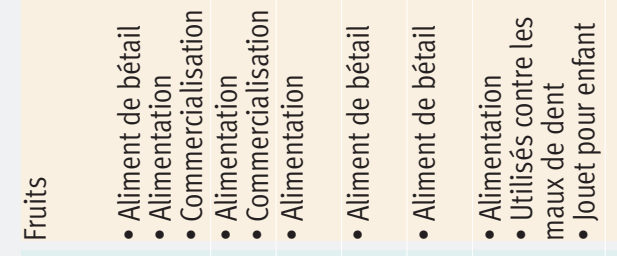

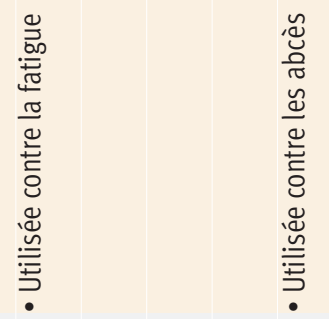

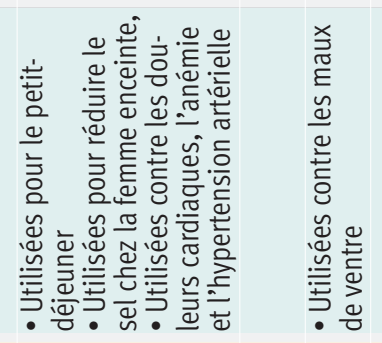

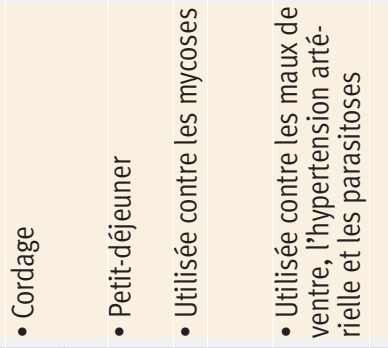
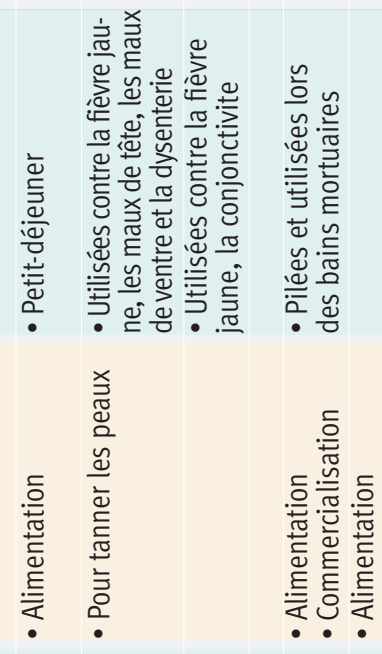

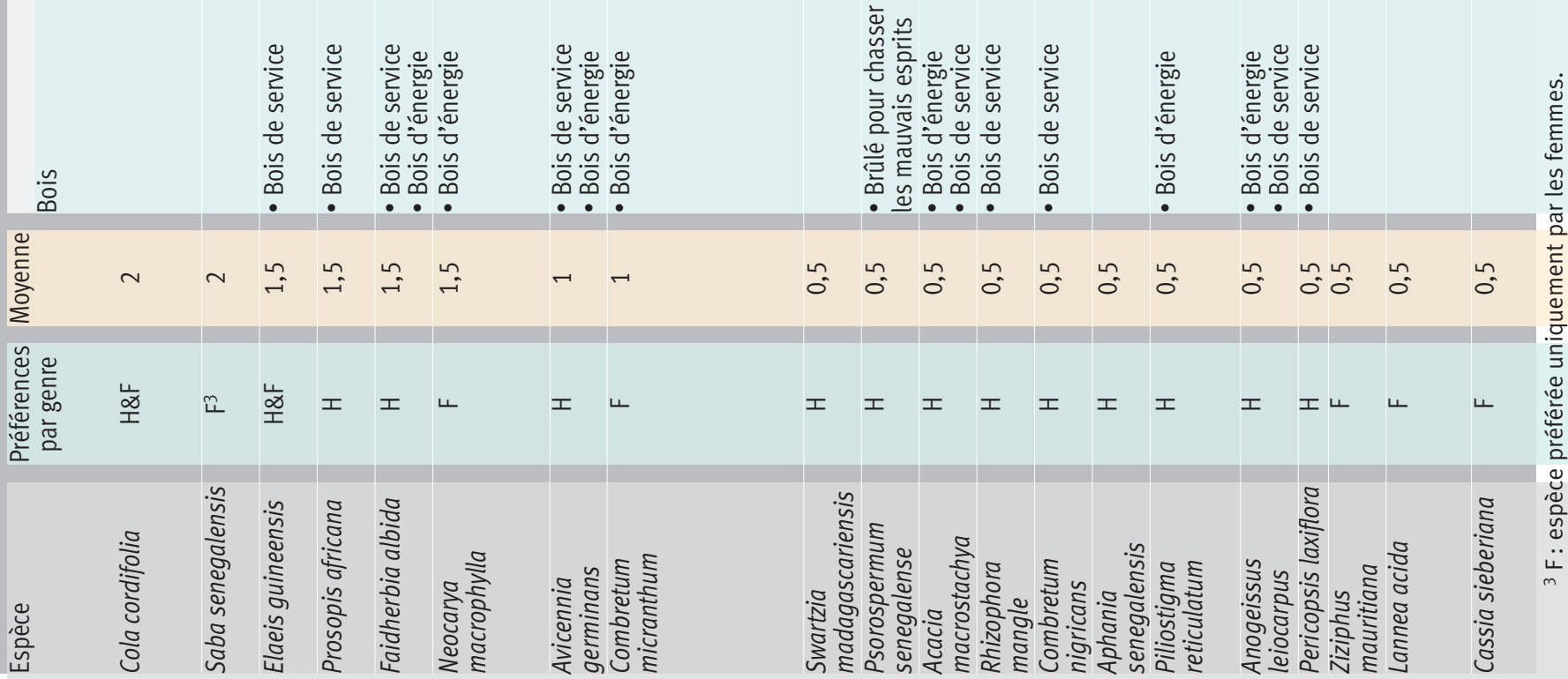




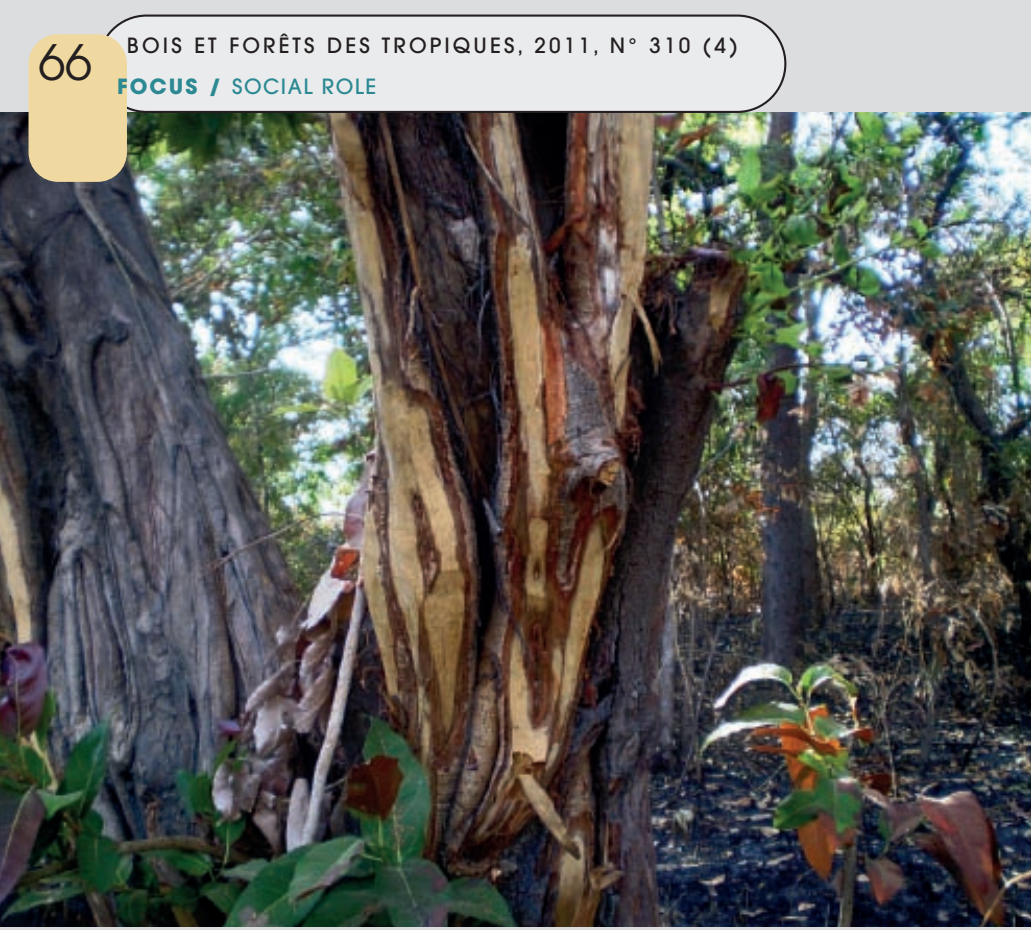

Photo 6.

Pied de Ficus iteophylla écorcé à l'intérieur de la forêt classée.

Photo M. Diop.

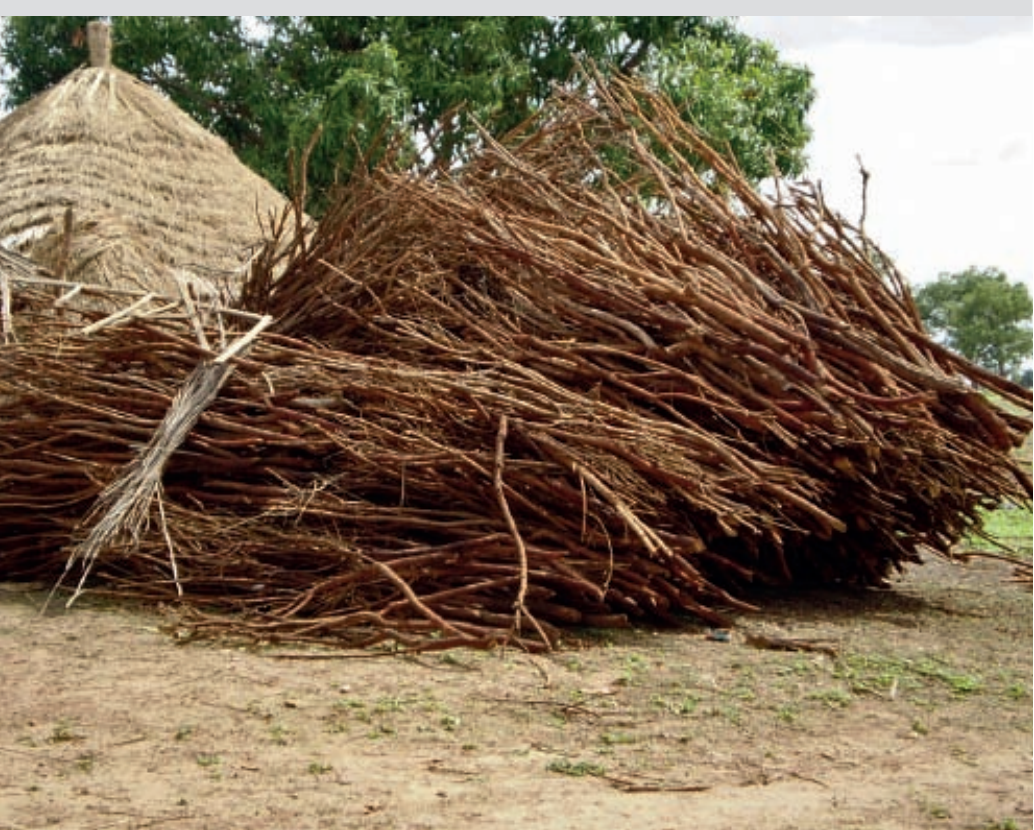

Photo 7.

Bois de Combretum glutinosum stocké à côté

d'une concession. Ce bois fait de plus en plus l'objet d'une commercialisation.

Photo M. Diop.

Le fait que les préférences des femmes soient marquées par une abondance des espèces à fruits comestibles semble s'inscrire dans une logique d'autosubsistance qui les affecte plus que les hommes. D'après la FAo (1989), en milieu rural africain, les femmes sont davantage tributaires que les hommes des arbres et des produits forestiers alimentaires. C'est le cas des femmes des localités périphériques de
Patako. Elles sont plus présentes dans la cueillette des fruits sauvages que les hommes. Ces fruits constituent ainsi un élément important du régime alimentaire, surtout pour les enfants. En plus de cela, ces fruits ont une fonction sociale très importante dans le raffermissement des liens avec leur utilisation comme cadeaux lors des déplacements en milieu urbain ou lorsque ces femmes reçoivent un hôte citadin. Dans un autre registre, la place prépondérante qu'occupent les espèces fruitières dans les classifications féminines résulte de leur contribution à l'économie féminine locale. La plupart des fruits sont commercialisés dans le village ou dans les luuma (marchés hebdomadaires traditionnels) et les revenus tirés permettent de satisfaire les besoins domestiques (généralement imposés par la pauvreté), tels que contribuer à la dépense quotidienne, participer aux tontines ou gérer les petites charges de fonctionnement du ménage. Au demeurant, ce rôle déterminant des fruits sauvages dans l'existence des femmes en milieu rural a été noté dans plusieurs pays africains (EASTON, RonALD, 2000), notamment chez les Kel Tamasheq du Niger (BERNUS, 1967), au Zimbabwe (CAMPBELL, 1987), en Zambie (PACKHAM, 1993), chez les Karugu de Tanzanie (THEILADE et al., 2007), chez les habitants de Dem et de Wédsé au Burkina Faso (BELEM et al., 2008), dans le Sud du Cameroun (LESCUYER, 2010). Leurs travaux ont démontré que ces fruits, plus qu'un simple complément alimentaire, permettent de compléter une ration parfois largement insuffisante durant les périodes de disette et de soudure.

Ce ne sont pas seulement les fruits sauvages qui interviennent dans l'économie féminine locale, mais aussi les écorces de Daniellia oliveri (photo 8) et les résines de Sterculia setigera. L'écorce de Daniellia oliveri intéresse particulièrement la plupart des femmes des localités riveraines de la forêt de Patako qui l'utilisent comme encens, ainsi que pour chasser les moustiques et les mauvais esprits.

Le nombre d'espèces préférées par les femmes est relativement moins important que celui des espèces préférées par les hommes (21 contre 28). Cela s'explique par le fait que les femmes ont des besoins en ressources végétales généralement concentrés sur trois préoccupations majeures : l'énergie, l'alimentation et la santé. Ainsi, toutes les ressources exploitées par ces dernières servent à la satisfaction de ces principaux besoins.

Parmi les espèces préférées par les riverains de la forêt, seulement Detarium microcarpum, Combretum glutinosum, Combretum nigricans et Pterocarpus erinaceus présentent une structure normale, encore que la régénération naturelle de Pterocarpus erinaceus soit relativement faible (SAmBou, 2004). Quant à Cordyla pinnata et Parkia biglobosa, qui sont les espèces de grande préférence, leurs populations présentent une structure nettement dégradée du fait surtout de problèmes de survie de la régénération naturelle (SAMBOU, 2004 ; NIANG-DIOP, 2005). Ces espèces, malgré leur importance socioéconomique, ne font pas l'objet d'une protection particulière de la part des populations riveraines. Hormis une sacralisation relative de Adansonia digitata, Khaya senegalensis et Tamarindus indica, les rares initiatives locales sont des interdictions de coupe en zone de terroir, se limitant essentiellement à Cordyla pinnata et Parkia biglobosa et très peu respectées surtout par les allochtones. 


\section{Conclusion}

Il apparaît que, en matière de ressources forestières végétales ligneuses, les préférences des hommes et des femmes des localités périphériques de la forêt classée de Patako sont influencées par les préoccupations quotidiennes, les rôles sociaux et les maladies les plus fréquentes dans cette zone. Si le bois de service a largement déterminé le choix des hommes, celui des femmes a été amplement influencé par les fruits sauvages, éléments essentiels dans l'alimentation et l'économie féminines locales. Au-delà des préférences des femmes et des hommes, le nombre d'espèces (34) retenues dans les choix traduit une certaine connaissance des ressources végétales de la forêt, avec aussi une large utilisation de ces dernières qui constituent, entre autres, un soutien financier important des villageois complétant la ration alimentaire et les revenus par du petit commerce très localisé. Toutefois, l'implication de personnes-ressources comme le chef de village et la présidente du groupement des femmes dans le choix des participants peut constituer une limite car, malgré la définition de critères, elle ne garantit pas une bonne représentativité des participants. En outre, les classifications préférentielles ont été faites en fonction du sexe uniquement, alors que l'âge et la catégorie socioprofessionnelle pouvaient être des variables de comparaison. Enfin, l'étude a porté uniquement sur les espèces ligneuses, alors que les espèces non ligneuses notamment les plantes de sous-bois jouent un rôle prépondérant en milieu rural et, de ce fait, doivent être préservées lors de la reprise en main de la gestion des forêts.
Cependant, le fait que Cordyla pinnata et Parkia biglobosa aient obtenu les scores les plus élevés dans les deux classifications préférentielles traduit toute leur importance pour les populations locales. D'où la nécessité pour tout programme ou projet de gestion des ressources forestières dans cette zone de leur accorder une attention particulière. Audelà de cette attention, qui ne doit pas se limiter à ces deux espèces, la connaissance des préférences doit aider (aux prises de décisions) et accompagner les différents acteurs de la gestion des ressources naturelles, pour un choix judicieux des espèces dans les activités de restauration du couvert végétal. Ainsi, au lieu de favoriser en premier les espèces exotiques, les gestionnaires doivent surtout accorder la priorité aux espèces locales préférées pour renforcer l'arbre dans les terroirs à travers des activités de plantation, d'agroforesterie et de régénération naturelle assistée. Cela dans le but de réduire la pression exercée sur la forêt classée et d'amoindrir les risques de pression démographique; sachant que dans les années à venir, la demande énergétique domestique croissante perturbera l'équilibre du milieu naturel actuel.

\section{Remerciements}

Ce travail a été réalisé grâce au Projet régional «Tools for Management and Sustainable Use of Natural Vegetation in West Africa " (EU FP6 031685). Nous remercions aussi toutes les bonnes volontés qui ont aidé à l'amélioration de cet article de par leurs observations, leurs contributions et leurs suggestions.

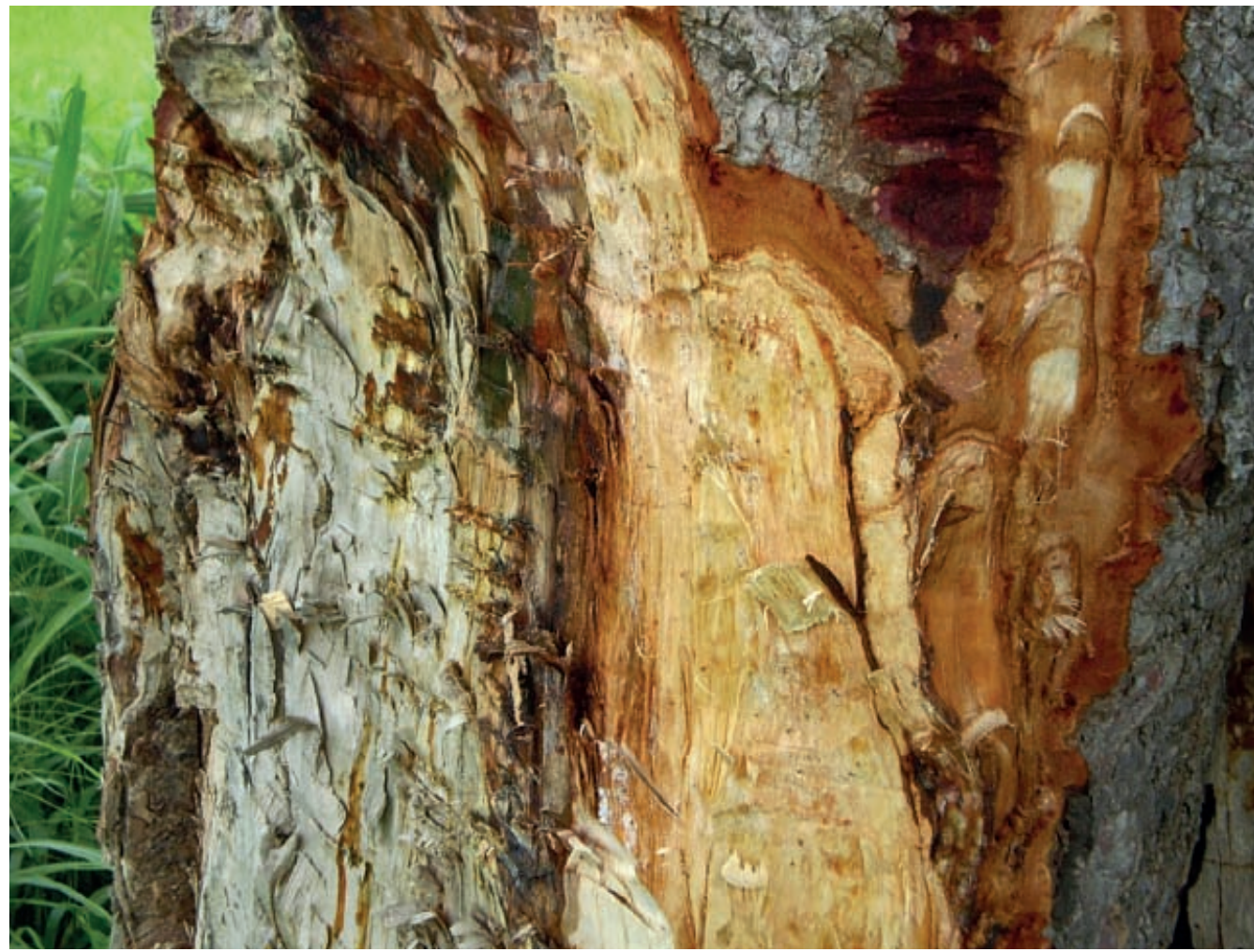

Photo 8.

Individu de Daniellia oliveri écorcé. Les écorces sont vendues dans les luuma (marchés hebdomadaires traditionnels) entre 100 et 300 francs Cfa le sachet. Photo M. Diop. 
ADAM J. G., 1970. Noms vernaculaires de plantes du Sénégal. Paris, France, Muséum national d'histoire naturelle, $112 \mathrm{p}$. ARIORI S. L., OZER P., 2005. Évolution des ressources forestières en Afrique de l'Ouest soudano-sahélienne au cours des 50 dernières années. Revue Internationale de Géologie, de Géographie et d’Écologie Tropicales, 29 : 61-68.

BELEM B., OLSEN C. S., THEILADE I., BELLEFONTAINE R., GUINKO S., LYKKE A. M., DIALLO A., BOUSSIM J. I., 2008. Identification des arbres hors forêt préférés des populations du Sanmatenga (Burkina Faso). Bois et Forêts des Tropiques, 298 (4) : 55-66.

BERGERET A., RIBOT J. C., 1990. L'arbre nourricier en pays sahélien. Paris, France, Éditions de la Maison des sciences de l'homme, $237 \mathrm{p}$.

BERHAUT J., 1967. Flore du Sénégal. Dakar, Sénégal, Clairafrique, $485 \mathrm{p}$.

BERNUS E., 1967. Cueillette et exploitation des ressources spontanées du sahel nigérien par les Kel Tamasheq. Cahiers Orstom, série Sciences humaines, IV (I) : 31-52.

BRONDEAU F., 2001. Évolution de la filière bois énergie et dynamique des formations ligneuses autour de l'Office du Niger. Bois et Forêts des Tropiques, 270 (4) : 15-33.

CAMPBELL B. M., 1987. The Use of Wild Fruits in Zimbabwe. Food and Nutrition, 12: 38-44.

COLLECTIF, 2007. Atlas du Sénégal. Paris, France, Éditions du Jaguar, $136 \mathrm{p}$.

COMPAGNON D., CONSTANTIN F., 2000. Administrer l'environnement en Afrique : gestion communautaire, conservation et développement durable. Paris, France, Karthala, 497 p.

DIOP M., KAYA B., NIANG A., OLIVIER A., 2005. Les espèces ligneuses et leurs usages : les préférences des paysans dans le Cercle de Ségou, au Mali. Nairobi, Kenya, World Agroforestry Centre (Icraf), Working Paper $n^{\circ}$ 9, 26 p.

EASTON P., RONALD M., 2000. Les femmes et la biodiversité végétale en Afrique. Washington, États-Unis, Banque mondiale, Notes CA n ${ }^{\circ} 23,4 \mathrm{p}$.

FAO, 1989. Rétablir l'équilibre. Les femmes et les ressources forestières. Fao, Asdi, 33 p.

FAO, 2005. Évaluation des ressources forestières mondiales 2005. Rapport principal. Rome, Fao, Étude Fao Forêts 147, 351 p.

GANABA S., OUADBA J. M., BOGNOUNOU O., 1998. Les ligneux à usage de bois d'énergie en région sahélienne du Burkina Faso : préférences des groupes ethniques. Sécheresse, 9 (4) : 261-268.

GANABA S., OUADBA J. M., BOGNOUNOU O., 2004. Plantes de construction d'habitations en région sahélienne. Bois et Forêts des Tropiques, 282 (4) : 11-17.

GAUTIER D., 1994. Valeur d'usage de l'arbre en pays bamiléké. Bois et Forêts des Tropiques, 241 : 40-50.

GUEYE B., FREUDENBERGER K. S., 1991. Introduction à la méthode accélérée de recherche participative (MARP). Rapid Rural Appraisal. Quelques notes pour appuyer une formation pratique. Buenos Aires, Argentine, International Institute for Environment Development (lied), 70 p.
LAWANI A., 2007. Contribution du bois énergie aux moyens d'existence durables des ménages riverains de la Réserve de Biosphère de la Pendjari. Mémoire, université d’Abomey Calavi, Bénin, 133 p.

LESCUYER G., 2010. Importance économique des produits forestiers non ligneux dans quelques villages du Sud-Cameroun. Bois et Forêts des Tropiques, 304 (2) : 15-24.

NIANG-DIOP F., 2005. Étude de facteurs limitant la régénération naturelle de Bombax costatum, Cordyla pinnata, Parkia biglobosa, Prosopis africana et Pterocarpus erinaceus dans la forêt de Fathala (parc national du delta du Saloum, Sénégal). Thèse, université Cheikh Anta Diop, Sénégal, 122 p.

NOUVELLET Y., SYLLA M. L., KASSAMBARA A., 2003. La production de bois d'énergie dans les jachères au Mali. Bois et Forêts des Tropiques, 276 (2) : 5-15.

OUEDRAOGO B., 2007. Filière bois d'énergie burkinabé : structuration des prix et analyse de la répartition des bénéfices. Bois et Forêts des Tropiques, 294 (4) : 75-88.

PACKHAM J., 1993. Valeur des arbres fruitiers indigènes des régions boisées du Miombo du Centre de l'Afrique Australe. Document du Rural Development Forestry Network (Rdfn) $\mathrm{n}^{\circ} 15 \mathrm{c}, 16 \mathrm{p}$.

RÉPUBLIQUE DU SÉNÉGAL, 2005a. Politique forestière du Sénégal 2005-2025, document principal. Ministère de l’Environnement et de la Protection de la Nature, $156 \mathrm{p}$.

RÉPUBLIQUE DU SÉNÉGAL, 2005b. Rapport sur l'état de l'environnement au Sénégal. Ministère de l'Environnement et de la Protection de la Nature, Centre de suivi écologique, $230 \mathrm{p}$. RÉPUBLIQUE DU SÉNÉGAL, 2006a. Document de Stratégie pour la croissance et la réduction de la pauvreté 2006-2010. Gouvernement du Sénégal, Banque mondiale, 103 p.

RÉPUBLIQUE DU SÉNÉGAL, 2006b. Situation économique et sociale de la région de Fatick. Agence nationale de la statistique et de la démographie, 145 p.

SAMBOU B., 2004. Évaluation de l'état, de la dynamique et des tendances évolutives de la flore et de la végétation ligneuses dans les domaines soudanien et sub-guinéen au Sénégal. Thèse, université Cheikh Anta Diop, Sénégal, 210 p. SOW M., ANDERSON J., 1996. Perceptions and classification of woodhand by Malinké villagers near Bamako, Mali. Unasylva, 186.

THEILADE I., HANSEN H. H., KROG M., RUFFO C. K., 2007. Use values and relative importance of trees to the Kaguru people in semi-arid Tanzania. Part II: Woodland species. Forests, Trees and Livelihoods, 17: 109-123.

TOBITH C., CUNY P., 2006. Genre et foresterie communautaire au Cameroun. Quelles perspectives pour les femmes ? Bois et Forêts des Tropiques, 289 (3) : 17-26.

ZERBO P., MILLOGO-RASOLODIMBY J., NACOULMA-OUEDRAOGO O. G., VAN DAMME P., 2011. Plantes médicinales et pratiques médicales au Burkina Faso : cas des Sanan. Bois et Forêts des Tropiques, 307 (1) : 41-53. 